J. Korean Math. Soc. 51 (2014), No. 5, pp. 1029-1044

http://dx.doi.org/10.4134/JKMS.2014.51.5.1029

\title{
A MULTIVARIABLE MAYER-ERDÖS PHENOMENON
}

\author{
Xianchang Meng and Alexandru Zaharescu
}

ABStRACT. In this paper we consider a generalization of the Mayer-Erdös phenomenon discussed in [12] to linear forms in a larger number of variables.

\section{Introduction}

Let $Q$ be a positive integer. The Farey series $\mathcal{F}_{Q}$ of order $Q$ consists of reduced fractions between 0 and 1 whose denominators do not exceed $Q$. For the basic properties of Farey series, the reader is referred to Hardy and Wright [9], Chapter 3. Let $N(Q)$ denote the number of elements of $\mathcal{F}_{Q}$. We write these elements in increasing order,

$$
\gamma_{1}=\frac{a_{1}}{q_{1}}<\gamma_{2}=\frac{a_{2}}{q_{2}}<\cdots<\gamma_{N(Q)}=\frac{a_{N(Q)}}{q_{N(Q)}} .
$$

Hardy, Littlewood and Pólya [8] proposed the concept of similar ordering for pairs of rational numbers, as follows. If all the reduced fractions $r=\frac{a}{q}$ and $r^{\prime}=\frac{a^{\prime}}{q^{\prime}}$ satisfy

$$
\left(a-a^{\prime}\right)\left(q-q^{\prime}\right) \geq 0,
$$

then $r$ and $r^{\prime}$ are said to be similarly ordered.

Mayer [10] proved that the first and second neighbors in any Farey series are similarly ordered, and he also proved the similar ordering of the third neighbors with the exception of $\mathcal{F}_{4}$. Hardy suggested and Mayer [11] confirmed that the $k$-th neighbors are similarly ordered, with a finite number (depending only on $k$ ) of exceptions. Mayer showed the following:

Given a positive integer $k$, there exists a number $Q(k)$ such that for any $Q>Q(k)$, and any $1 \leq j<j^{\prime} \leq N(Q)$ with $j^{\prime}-j \leq k$, the numbers $\frac{a_{j}}{q_{j}}$ and $\frac{a_{j^{\prime}}}{q_{j^{\prime}}}$ are similarly ordered.

Erdös [7] established a vast improvement of Mayer's result:

Received February 5, 2014; Revised June 11, 2014.

2010 Mathematics Subject Classification. 11B57.

Key words and phrases. farey fractions, similar ordering, Mayer-Erdös phenomenon. 
There exists an absolute constant $C>0$ such that, for any positive integer $Q$ and any $1 \leq j<j^{\prime} \leq N(Q)$ for which $j^{\prime}-j<\frac{Q}{C}$, the fractions $\frac{a_{j}}{q_{j}}$ and $\frac{a_{j^{\prime}}}{q_{j^{\prime}}}$ are similarly ordered.

One of the authors [12] extended the result of Erdös by considering a general linear form in two variables

$$
L(X, Y)=A X+B Y,
$$

and proving the following result:

There exists an absolute constant $C>0$ such that, for any positive integers $D, Q$, any integer numbers $|A|,|B| \leq D$, and any $1 \leq j<j^{\prime} \leq N(Q)$ satisfying the inequality $j^{\prime}-j \leq \frac{Q}{C D^{2}}$, we have

$$
\left(A a_{j}+B a_{j^{\prime}}\right)\left(A q_{j}+B q_{j^{\prime}}\right) \geq 0 .
$$

Note that the result by Erdös in [7] is for the case $D=1$.

In the present paper, our goal is to extend the results further by considering a general linear form in $v$ variables. Let $v \geq 3$ be an integer and let $D$ be a positive integer. Let $\left\{A_{i}\right\}_{i=1}^{v}$ be integers with $\left|A_{i}\right| \leq D$ for all $1 \leq i \leq v$. Consider the linear form,

$$
L(\mathbf{x}):=\sum_{i=1}^{v} A_{i} x_{i}=A_{1} x_{1}+A_{2} x_{2}+\cdots+A_{v} x_{v},
$$

where $\mathbf{x}:=\left(x_{1}, x_{2}, \ldots, x_{v}\right)$.

For any $v$ elements in the Farey series of order $Q$,

$$
\frac{a_{r_{1}}}{q_{r_{2}}}, \frac{a_{r_{2}}}{q_{r_{2}}}, \ldots, \frac{a_{r_{v}}}{q_{r_{v}}},
$$

where $1 \leq r_{i} \leq N(Q)(1 \leq i \leq v)$, we let $\mathbf{a}:=\left(a_{r_{1}}, a_{r_{2}}, \ldots, a_{r_{v}}\right)$ and $\mathbf{q}:=$ $\left(q_{r_{1}}, q_{r_{2}}, \ldots, q_{r_{v}}\right)$. Then, we have the following result:

Theorem 1. There exists a positive constant $C=C(v)$ which only depends on $v$ such that, for any positive integers $D$ and $Q$, any set of integers $\left\{A_{i}\right\}_{i=1}^{v}$ with $\left|A_{i}\right| \leq D$ satisfying:

1) $\sum_{i=1}^{v} A_{i} \neq 0$, and

2) $\sum_{i \neq k} A_{i} \neq 0$ for any $1 \leq k \leq v$,

and for any $v$-tuple $1 \leq r_{1}<r_{2}<\cdots<r_{v} \leq N(Q)$, with

we have

$$
r_{v}-r_{1} \leq \frac{Q}{C D^{2}}
$$

$$
L(\mathbf{a}) L(\mathbf{q})=\left(\sum_{i=1}^{v} A_{i} a_{r_{i}}\right)\left(\sum_{i=1}^{v} A_{i} q_{r_{i}}\right) \geq 0 .
$$


Remark 1. If we let $d=\sum_{i=1}^{v} A_{i}$, the conditions 1) and 2) are equivalent to: $d \neq 0$ and $d \notin\left\{A_{i}\right\}_{i=1}^{v}$. One can use this statement to check if the $A_{i}$ 's satisfy the conditions from the statement of the theorem in concrete numerical examples. In our proof, the conditions 1) and 2) are more convenient to use.

Remark 2. Surprisingly, as we shall see below, for $v \geq 3$, counterexamples do exist if one does not assume any one of the above two conditions. See Section 3 for the details.

We end this section with a discussion of a geometric interpretation of the Mayer-Erdös phenomenon. There is a natural one-to-one correspondence between the set of Farey fractions of order $Q$ and the set of visible lattice points inside the triangle with vertices $(0,0),(Q, 0),(Q, Q)$, where a visible point $(q, a)$ corresponds to the Farey fraction $a / q$. In this correspondence, Farey fractions are arranged in increasing order, and the visible points are arranged in increasing order of the slope of the ray from the origin that passes through them. Various problems where visible points play an important role have been studied by a number of authors, see for example [1], [2], [3], [4], [5], [6], and the references therein. A peculiar geometric aspect of the Mayer-Erdös phenomenon, which to our knowledge has not been exploited so far, is the following. Consider, for any visible point $(q, a)$ the coordinate system with horizontal and vertical axes parallel to the initial ones, centered at the point $(q, a)$. Then, by the theorem of Erdös mentioned earlier, the first about a constant times $Q$ many visible points following $(q, a)$ lie inside the first or third quadrant. The same holds for the previous about a constant times $Q$ many visible points which appear before $(q, a)$ in above ordering. The generalizations of Erdös' theorem obtained in [12] and in the present paper have similar geometric interpretations.

\section{Proof of Theorem 1}

If the $A_{i}$ 's are all positive or all negative, the result is trivial. Let's assume that at least one of them is positive, and at least one of them is negative. Let $M$ be the number of positive coefficients, and $N$ be the number of negative coefficients, so $M+N=v$. We collect all the positive coefficients together, and all the negative coefficients together. We then rearrange the indices, let $s_{j}=r_{M+j}$, and write

$$
L(\mathbf{a}) L(\mathbf{q})=\left(\sum_{i=1}^{M} A_{i} a_{r_{i}}-\sum_{j=1}^{N} B_{j} a_{s_{j}}\right)\left(\sum_{i=1}^{M} A_{i} q_{r_{i}}-\sum_{j=1}^{N} B_{j} q_{s_{j}}\right),
$$

where $A_{1}, \ldots, A_{M}, B_{1}, \ldots, B_{N}$ are all positive integers.

Let us suppose that $L(\mathbf{a}) L(\mathbf{q})<0$. Let $r=\min \left\{r_{i}: 1 \leq i \leq v\right\}$ and $s=\max \left\{r_{i}: 1 \leq i \leq v\right\}$. We want to show that $s-r>\frac{Q}{C D^{2}}$ for some constant $C=C(v)$. We distinguish two cases: 
Case I:

$$
\left\{\begin{array}{l}
\sum_{i=1}^{M} A_{i} a_{r_{i}}-\sum_{j=1}^{N} B_{j} a_{s_{j}} \leq-1 \\
\sum_{i=1}^{M} A_{i} q_{r_{i}}-\sum_{j=1}^{N} B_{j} q_{s_{j}} \geq 1 .
\end{array}\right.
$$

\section{Case II:}

Denote

$$
\left\{\begin{array}{l}
\sum_{i=1}^{M} A_{i} a_{r_{i}}-\sum_{j=1}^{N} B_{j} a_{s_{j}} \geq 1 \\
\sum_{i=1}^{M} A_{i} q_{r_{i}}-\sum_{j=1}^{N} B_{j} q_{s_{j}} \leq-1 .
\end{array}\right.
$$

and

$$
\Delta\left(r_{i}, s_{j}\right):=\frac{q_{r_{i}} a_{s_{j}}-a_{r_{i}} q_{s_{j}}}{q_{r_{i}} q_{s_{j}}}=\frac{a_{s_{j}}}{q_{s_{j}}}-\frac{a_{r_{i}}}{q_{r_{i}}}
$$

$$
\Delta:=\frac{a_{s}}{q_{s}}-\frac{a_{r}}{q_{r}}
$$

where $r=\min \left\{r_{1}, \ldots, r_{M}, s_{1}, \ldots, s_{N}\right\}$, and $s=\max \left\{r_{1}, \ldots, r_{M}, s_{1}, \ldots, s_{N}\right\}$.

We need an estimate for $\Delta$.

\subsection{A lower bound for $\Delta$}

We deal with Case I first. We have

$$
\sum_{i=1}^{M} A_{i} q_{r_{i}} \geq \sum_{j=1}^{N} B_{j} q_{s_{j}}+1
$$

and

$$
\sum_{j=1}^{N} B_{j} a_{s_{j}} \geq \sum_{i=1}^{M} A_{i} a_{r_{i}}+1
$$

Multiplying both sides of the above two inequalities, one has

$$
\left(\sum_{i=1}^{M} A_{i} q_{r_{i}}\right)\left(\sum_{j=1}^{N} B_{j} a_{s_{j}}\right) \geq\left(\sum_{j=1}^{N} B_{j} q_{s_{j}}+1\right)\left(\sum_{i=1}^{M} A_{i} a_{r_{i}}+1\right) .
$$

Then,

$$
\sum_{i=1}^{M} \sum_{j=1}^{N} A_{i} B_{j}\left(q_{r_{i}} a_{s_{j}}-a_{r_{i}} q_{s_{j}}\right) \geq \sum_{i=1}^{M} A_{i} a_{r_{i}}+\sum_{j=1}^{N} B_{j} q_{s_{j}}+1 .
$$

We divide by the product $\prod_{i, j} A_{i} B_{j} q_{r_{i}} q_{s_{j}}$ on both sides of (2.1) to obtain

$$
\sum_{i=1}^{M} \sum_{j=1}^{N} \frac{\Delta\left(r_{i}, s_{j}\right)}{\prod_{k \neq i, l \neq j} A_{k} B_{l} q_{r_{k}} q_{s_{l}}}>\sum_{i=1}^{M} \frac{\gamma_{r_{i}}}{\prod_{k \neq i, l} A_{k} B_{l} q_{r_{k}} q_{s_{l}}}+\sum_{j=1}^{N} \frac{1}{\prod_{k, l \neq j} A_{k} B_{l} q_{r_{k}} q_{s_{l}}}
$$




$$
:=S_{1}+S_{2},
$$

where $\gamma_{r_{i}}=\frac{a_{r_{i}}}{q_{r_{i}}}$, and $S_{1}$ and $S_{2}$ denote the first and respectively the second sum on the right side above. Let

$$
S=\sum_{i=1}^{M} \sum_{j=1}^{N} \frac{1}{\prod_{k \neq i, l \neq j} A_{k} B_{l} q_{r_{k}} q_{s_{l}}} .
$$

Since $\Delta\left(r_{i}, s_{j}\right) \leq \Delta$ for any $i, j$, we have $\Delta \cdot S>S_{1}+S_{2}$, so

$$
\Delta>\frac{S_{1}+S_{2}}{S} \text {. }
$$

For each $1 \leq i \leq M$,

$$
S_{2}=\sum_{j=1}^{N} \frac{1}{\prod_{k, l \neq j} A_{k} B_{l} q_{r_{k}} q_{s_{l}}} \geq \frac{1}{D Q} \sum_{j=1}^{N} \frac{1}{\prod_{k \neq i, l \neq j} A_{k} B_{l} q_{r_{k}} q_{s_{l}}} .
$$

We sum over $i$ to obtain

$$
M S_{2} \geq \frac{1}{D Q} \sum_{i=1}^{M} \sum_{j=1}^{N} \frac{1}{\prod_{k \neq i, l \neq j} A_{k} B_{l} q_{r_{k}} q_{s_{l}}}=\frac{1}{D Q} S .
$$

Thus,

$$
\frac{S_{2}}{S} \geq \frac{1}{D Q M}
$$

Similarly, for each $1 \leq j \leq N$,

$$
S_{1}=\sum_{i=1}^{M} \frac{\gamma_{r_{i}}}{\prod_{k \neq i, l} A_{k} B_{l} q_{r_{k}} q_{s_{l}}} \geq \frac{1}{D Q} \sum_{i=1}^{M} \frac{\gamma_{r_{i}}}{\prod_{k \neq i, l \neq j} A_{k} B_{l} q_{r_{k}} q_{s_{l}}},
$$

and summing over $j$, we have

$$
\begin{aligned}
N S_{1} & \geq \frac{1}{D Q} \sum_{i=1}^{M} \sum_{j=1}^{N} \frac{\gamma_{r_{i}}}{\prod_{k \neq i, l \neq j} A_{k} B_{l} q_{r_{k}} q_{s_{l}}} \\
& :=\frac{1}{D Q} S_{\gamma} .
\end{aligned}
$$

So $S_{1} \geq \frac{1}{D Q N} S_{\gamma}$, and

$$
\frac{S_{1}}{S} \geq \frac{1}{D Q N} \frac{S_{\gamma}}{S}=\frac{1}{D Q N} \tilde{\gamma},
$$

where $\tilde{\gamma}=\frac{S_{\gamma}}{S}$. Since $\gamma_{r_{i}}<1, S_{\gamma}<S$, so $0<\tilde{\gamma}<1$.

Combining (2.2), (2.3) and (2.4), we obtain

$$
\Delta>\frac{1}{D Q}\left(\frac{1}{M}+\frac{\tilde{\gamma}}{N}\right) .
$$


For Case II, we only need to change the positions of $a_{r_{i}}$ and $q_{r_{i}}$, and change the positions of $a_{s_{j}}$ and $q_{s_{j}}$. The calculations are similar to those in Case I. We find that

$$
\Delta>\frac{1}{D Q}\left(\frac{1}{N}+\frac{\tilde{\gamma}^{\prime}}{M}\right)
$$

where

$$
\tilde{\gamma}^{\prime}=\frac{S_{\gamma^{\prime}}}{S}, \quad S_{\gamma^{\prime}}=\sum_{i=1}^{M} \sum_{j=1}^{N} \frac{\gamma_{s_{j}}}{\prod_{k \neq i, l \neq j} A_{k} B_{l} q_{r_{k}} q_{s_{l}}}, \quad \gamma_{s_{j}}=\frac{a_{s_{j}}}{q_{s_{j}}},
$$

and by the definition of $S$ we see that $0<\tilde{\gamma}^{\prime}<1$.

Hence, combining (2.5) and (2.6), in all cases we have,

$$
\Delta>\frac{1}{D Q(M+N)}=\frac{1}{v D Q} .
$$

\subsection{An upper bound for $\Delta$}

Let $r=\min \left\{r_{1}, r_{2}, \ldots, r_{v}\right\}$, and $s=\max \left\{r_{1}, r_{2}, \ldots, r_{v}\right\}$. We have

$$
\Delta=\frac{a_{s}}{q_{s}}-\frac{a_{r}}{q_{r}}=\sum_{j=r+1}^{s}\left(\frac{a_{j}}{q_{j}}-\frac{a_{j-1}}{q_{j-1}}\right)=\sum_{j=r+1}^{s} \frac{1}{q_{j} q_{j-1}} .
$$

Here, in the last equality, we used the property of Farey neighbors which states that $a_{j} q_{j-1}-a_{j-1} q_{j}=1$.

Denote by $j^{*}$ the smallest element of the set $\{r, r+1, \ldots, s\}$ for which the equality

$$
q_{j^{*}}=\min \left\{q_{j}: r \leq j \leq s\right\}
$$

holds. In [12], part of the proof is independent of the linear form. We apply that method to deal with some of our cases. Let $T \geq 1$ be a parameter, whose size will be determined later, and denote by $r_{1}^{\prime}, \ldots, r_{t}^{\prime}$ those elements from the set $\{r, r+1, \ldots, s\}$ for which

$$
q_{r_{1}^{\prime}}, q_{r_{2}^{\prime}}, \ldots, q_{r_{t}^{\prime}} \leq \frac{Q}{T}
$$

We arrange the above numbers in increasing order, $r_{1}^{\prime}<r_{2}^{\prime}<\cdots<r_{t}^{\prime}$. Next, we distinguish several cases.

For the cases $t=0$ and $t \geq 2$, we apply the method from [12].

If $t=0$, then $q_{r}, q_{r+1}, \ldots, q_{s}>\frac{Q}{T}$, and since $\max \left\{q_{j}, q_{j-1}\right\} \geq \frac{q_{j}+q_{j-1}}{2}$, we have

$$
\frac{1}{q_{j} q_{j-1}} \leq \frac{2}{\left(q_{j}+q_{j-1}\right) \min \left\{q_{j}, q_{j-1}\right\}}<\frac{2}{Q \min \left\{q_{j}, q_{j-1}\right\}} .
$$

Using (2.8) together with the above inequalities, we find that

$$
\Delta \leq \frac{2 T}{Q^{2}}(s-r)
$$


By (2.7), it follows in this case that

$$
s-r>\frac{Q}{2 v T D} .
$$

If $t=1$, then $r_{1}^{\prime}=j^{*}$, and by (2.11) of [12],

$$
\Delta \leq \frac{2}{q_{j^{*}} \max \left\{q_{j^{*}}, Q-q_{j^{*}}\right\}}+\frac{2 T(s-r-1)}{Q^{2}} .
$$

If $t \geq 2$, assume $T>8$, and then by (2.19) of [12], we have

$$
\Delta \cdot \frac{Q}{2}\left(1-\frac{8}{T}\right) \leq \frac{(s-r) T}{Q} .
$$

Using this formula and (2.7), we derive that

$$
s-r>\frac{Q}{2 v T D}\left(1-\frac{8}{T}\right) .
$$

By (2.9), (2.10) and (2.11), the proof of the theorem is finished in cases $t=0$ and $t \geq 2$ if we let $T$ be a suitable constant. Moreover, case $t=1$ is also done provided that $q_{j^{*}}$ is not very small.

\subsection{Case $t=1$}

In the following, we deal with the case $t=1$. Let $T^{\prime} \geq 1$ be another parameter, and assume that $q_{j^{*}} \geq T^{\prime} D$. By (2.21) in [12],

$$
\frac{2}{q_{j^{*}} \max \left\{q_{j^{*}}, Q-q_{j^{*}}\right\}} \leq \frac{2 T}{T^{\prime}(T-1) D Q} .
$$

Combining the above inequality, (2.7), and (2.10), we have

$$
\frac{2 T(s-r)}{Q^{2}} \geq \frac{1}{v D Q}-\frac{2 T}{T^{\prime}(T-1) D Q}=\frac{1}{D Q}\left(\frac{1}{v}-\frac{2 T}{T^{\prime}(T-1)}\right) .
$$

Then,

$$
s-r>\frac{Q}{D}\left(\frac{1}{2 v T}-\frac{1}{T^{\prime}(T-1)}\right) .
$$

We now assume that $q_{j^{*}}<T^{\prime} D$. Consider the neighbors of $\frac{a_{j^{*}}}{q_{j^{*}}}$ in the set of Farey fractions of order $Q$ with

$$
\frac{u}{v}<\frac{a_{j^{*}}}{q_{j^{*}}}<\frac{z}{w}
$$

By the argument in [12], we know that the first right neighbors of $\frac{z}{w}$ are

$$
\frac{z-k a_{j^{*}}}{w-k q_{j^{*}}}, \quad k=0,1,2, \ldots,\left[\frac{Q+1}{2 q_{j^{*}}}\right]-1,
$$

and the first left neighbors of $\frac{u}{v}$ are

$$
\frac{u-k^{\prime} a_{j^{*}}}{v-k^{\prime} q_{j^{*}}}, \quad k^{\prime}=0,1,2, \ldots,\left[\frac{Q+1}{2 q_{j^{*}}}\right]-1 .
$$


If $\frac{a_{j^{*}}}{q_{j^{*}}}$ does not coincide with $\frac{a_{r}}{q_{r}}$, and $\frac{a_{r}}{q_{r}}$ does not coincide with any of the fractions from $(2.14)$, then all these fractions lie between $\frac{a_{r}}{q_{r}}$ and $\frac{a_{s}}{q_{s}}$. We have

$$
s-r \geq\left[\frac{Q+1}{2 q_{j^{*}}}\right] \geq\left[\frac{Q+1}{2 T^{\prime} D}\right],
$$

in this case, the conclusion of the theorem is satisfied. Similarly, if $\frac{a_{j^{*}}}{q_{j^{*}}}$ does not coincide with $\frac{a_{s}}{q_{s}}$, and $\frac{a_{s}}{q_{s}}$ does not coincide with any fractions from (2.13), then again, all these fractions lie between $\frac{a_{r}}{q_{r}}$ and $\frac{a_{s}}{q_{s}}$. We obtain the same result as above.

Next we assume that $\frac{a_{r}}{q_{r}}$ coincides with $\frac{a_{j *}}{q_{j *}}$ or one of the fractions from (2.14), and $\frac{a_{s}}{q_{s}}$ coincides with $\frac{a_{j *}}{q_{j *}}$ or one of the fractions from (2.13). There are two cases:

1) $\frac{a_{j^{*}}}{q_{j^{*}}} \notin\left\{\frac{a_{r_{i}}}{q_{r_{i}}}: 1 \leq i \leq v\right\} \quad$ or $\quad$ 2) $\frac{a_{j^{*}}}{q_{j^{*}}} \in\left\{\frac{a_{r_{i}}}{q_{r_{i}}}: 1 \leq i \leq v\right\}$.

2.3.1. Case 1). If $\frac{a_{j^{*}}}{q_{j^{*}}} \notin\left\{\frac{a_{r_{i}}}{q_{r_{i}}}: 1 \leq i \leq v\right\}$, then each fraction $\frac{a_{r_{i}}}{q_{r_{i}}}(1 \leq i \leq v)$ has the form

$$
\frac{z-k_{i} a_{j^{*}}}{w-k_{i} q_{j^{*}}} \text { or } \frac{u-k_{i} a_{j^{*}}}{v-k_{i} q_{j^{*}}}
$$

for some integer $k_{i} \geq 0$. So, $a_{r_{i}}$ has the form

$$
z-k_{i} a_{j^{*}} \text { or } u-k_{i} a_{j^{*}}
$$

and $q_{r_{i}}$ has the form

$$
w-k_{i} q_{j^{*}} \text { or } v-k_{i} q_{j^{*}} .
$$

In the following, we want to estimate the size of $a_{r_{i}}$ and $q_{r_{i}}$.

Since $w+q_{j^{*}}>Q, v+q_{j^{*}}>Q$ (see [9], Chapter 3), and by the assumption $q_{j^{*}}<T^{\prime} D$, we have

$$
w-k_{i} q_{j^{*}}>Q-q_{j^{*}}-k_{i} q_{j^{*}}>Q-\left(k_{i}+1\right) T^{\prime} D .
$$

Similarly,

$$
v-k_{i} q_{j^{*}}>Q-q_{j^{*}}-k_{i} q_{j^{*}}>Q-\left(k_{i}+1\right) T^{\prime} D .
$$

So, we obtain, for any $1 \leq i \leq v$,

$$
q_{r_{i}}>Q-\left(k_{i}+1\right) T^{\prime} D .
$$

Let $\gamma=\frac{a_{j^{*}}}{q_{j^{*}}}$. We know that

$$
\frac{u}{v}<\frac{u+a_{j^{*}}}{v+q_{j^{*}}}<\frac{a_{j^{*}}}{q_{j^{*}}}<\frac{z+a_{j^{*}}}{w+q_{j^{*}}}<\frac{z}{w} .
$$

Then,

Therefore

$$
\frac{a_{j^{*}}}{q_{j^{*}}}-\frac{u+a_{j^{*}}}{v+q_{j^{*}}}=\frac{1}{q_{j^{*}}\left(v+q_{j^{*}}\right)}<\frac{1}{Q} .
$$

$$
u+a_{j^{*}}>\left(v+q_{j^{*}}\right)\left(\gamma-\frac{1}{Q}\right)>Q\left(\gamma-\frac{1}{Q}\right)=Q \gamma-1,
$$


and so

$$
u>Q \gamma-a_{j^{*}}-1
$$

By (2.17),

$$
\frac{z+a_{j^{*}}}{w+q_{j^{*}}}>\gamma
$$

so we have

$$
z>\left(w+q_{j^{*}}\right) \gamma-a_{j^{*}}>Q \gamma-a_{j^{*}} .
$$

Thus, by (2.18) and (2.19), for any $1 \leq i \leq v$, we obtain

(2.20) $a_{r_{i}}>Q \gamma-a_{j^{*}}-1-k_{i} a_{j^{*}}=Q \gamma-\left(k_{i}+1\right) \gamma q_{j^{*}}-1>Q \gamma-\left(k_{i}+1\right) \gamma T^{\prime} D-1$.

We also need an upper bound for $a_{r_{i}}$. Since

$$
\frac{a_{j^{*}}}{q_{j^{*}}}-\frac{u}{v}=\frac{1}{v q_{j^{*}}}
$$

we have

and

$$
u=v\left(\gamma-\frac{1}{v q_{j^{*}}}\right)=v \gamma-\frac{1}{q_{j^{*}}}<Q \gamma
$$

so,

$$
\frac{z}{w}-\frac{a_{j^{*}}}{q_{j^{*}}}=\frac{1}{w q_{j^{*}}}
$$

Thus, for any $1 \leq i \leq v$,

$$
z=w\left(\gamma+\frac{1}{w q_{j^{*}}}\right)=w \gamma+\frac{1}{q_{j^{*}}}<Q \gamma+1 .
$$

$$
a_{r_{i}}<Q \gamma+1
$$

In Case I, by condition 1), we have

$$
\sum_{i=1}^{M} A_{i} \neq \sum_{j=1}^{N} B_{j}
$$

If $\sum_{i=1}^{M} A_{i}<\sum_{j=1}^{N} B_{j}$, by (2.16), and the second inequality in Case I, we obtain

$$
\begin{aligned}
\left(\sum_{i=1}^{M} A_{i}\right) Q & >\sum_{i=1}^{M} A_{i} q_{r_{i}} \geq \sum_{j=1}^{N} B_{j} q_{s_{j}}+1>\sum_{j=1}^{N} B_{j}\left(Q-\left(k_{j}+1\right) T^{\prime} D\right) \\
& \geq\left(1+\sum_{i=1}^{M} A_{i}\right) Q-T^{\prime} D^{2} \sum_{j=1}^{N}\left(k_{j}+1\right)
\end{aligned}
$$

Therefore

$$
\sum_{j=1}^{N}\left(k_{j}+1\right)>\frac{Q}{T^{\prime} D^{2}} .
$$


If $\sum_{i=1}^{M} A_{i}>\sum_{j=1}^{N} B_{j}$, we use the first inequality in Case I, and by $(2.20)$ and (2.21), we have

$$
\begin{aligned}
\sum_{j=1}^{N} B_{j}(Q \gamma+1) & >\sum_{j=1}^{N} B_{j} a_{s_{j}} \geq \sum_{i=1}^{M} A_{i} a_{r_{i}}+1 \\
& >\sum_{i=1}^{M} A_{i}\left(Q \gamma-\left(k_{i}+1\right) \gamma T^{\prime} D-1\right) \\
& >Q \gamma\left(\sum_{i=1}^{M} A_{i}\right)-\gamma T^{\prime} D^{2} \sum_{i=1}^{M}\left(k_{i}+1\right)-\sum_{i=1}^{M} A_{i} \\
& \geq Q \gamma\left(1+\sum_{j=1}^{N} B_{j}\right)-\gamma T^{\prime} D^{2} \sum_{i=1}^{M}\left(k_{i}+1\right)-\sum_{i=1}^{M} A_{i}
\end{aligned}
$$

Then,

$$
T^{\prime} D^{2} \sum_{i=1}^{M}\left(k_{i}+1\right)+\frac{1}{\gamma}\left(\sum_{i=1}^{M} A_{i}+\sum_{j=1}^{N} B_{j}\right)>Q .
$$

Since $\gamma=\frac{a_{j^{*}}}{q_{j^{*}}}>\frac{1}{T^{\prime} D}, \frac{1}{\gamma}<T^{\prime} D$, we have

$$
\frac{1}{\gamma}\left(\sum_{i=1}^{M} A_{i}+\sum_{j=1}^{N} B_{j}\right)<T^{\prime} D^{2}(M+N)=T^{\prime} D^{2} v .
$$

It follows that

$$
\sum_{i=1}^{M}\left(k_{i}+1\right)+v>\frac{Q}{T^{\prime} D^{2}} .
$$

For Case II, in the above calculations, we only need to change the positions of $a_{r_{i}}$ and $q_{r_{i}}$, and change the positions of $a_{s_{j}}$ and $q_{s_{j}}$.

If $\sum_{i=1}^{M} A_{i}<\sum_{j=1}^{N} B_{j}$, by (2.20), (2.21), and the first inequality in Case II, we obtain

$$
\sum_{j=1}^{N}\left(k_{j}+1\right)+v>\frac{Q}{T^{\prime} D^{2}}
$$

If $\sum_{i=1}^{M} A_{i}>\sum_{j=1}^{N} B_{j}$, by (2.16), and the second inequality in Case II, we have

$$
\sum_{i=1}^{M}\left(k_{i}+1\right)>\frac{Q}{T^{\prime} D^{2}}
$$

By the definitions of $k_{i}$ and $k_{j}$, we can see that

$$
s-r \geq \max \left\{k_{i}, k_{j}: 1 \leq i \leq M, 1 \leq j \leq N\right\}+1 .
$$


Hence, combining (2.22), (2.24), (2.25), and (2.26), we obtain

$$
s-r>\frac{Q}{v T^{\prime} D^{2}}-1
$$

and since $s-r$ is an integer, one has

$$
s-r \geq\left[\frac{Q}{v T^{\prime} D^{2}}\right] \text {, if } \frac{a_{j^{*}}}{q_{j^{*}}} \notin\left\{\frac{a_{r_{i}}}{q_{r_{i}}}: 1 \leq i \leq v\right\} .
$$

2.3.2. Case 2). Next, we consider the case $\frac{a_{j^{*}}}{q_{j^{*}}} \in\left\{\frac{a_{r_{i}}}{q_{r_{i}}}: 1 \leq i \leq v\right\}$ which can be divided into two subcases:

$$
\frac{a_{j^{*}}}{q_{j^{*}}} \in\left\{\frac{a_{r_{i}}}{q_{r_{i}}}: 1 \leq i \leq M\right\} \quad \text { or } \quad \frac{a_{j^{*}}}{q_{j^{*}}} \in\left\{\frac{a_{s_{j}}}{q_{s_{j}}}: 1 \leq j \leq N\right\} .
$$

Let $j^{\prime}$ be the index of the coefficient associated to $a_{j^{*}}$ and $q_{j^{*}}$.

1) $\frac{a_{j^{*}}}{q_{j^{*}}} \in\left\{\frac{a_{r_{i}}}{q_{r_{i}}}: 1 \leq i \leq M\right\}$. By condition 2), we may assume that $\sum_{i \neq j^{\prime}} A_{i}$ $\neq \sum_{j=1}^{N} B_{j}$.

In Case I, if $\sum_{i \neq j^{\prime}} A_{i}<\sum_{j=1}^{N} B_{j}$, we use the second inequality in Case I, and (2.16), to obtain

$$
\begin{aligned}
\left(\sum_{i \neq j^{\prime}} A_{i}\right) Q+T^{\prime} D^{2} & >\sum_{i \neq j^{\prime}} A_{i} q_{r_{i}}+A_{j^{\prime}} q_{j^{*}} \geq \sum_{j=1}^{N} B_{j} q_{s_{j}}+1 \\
& >\sum_{j=1}^{N} B_{j}\left(Q-\left(k_{j}+1\right) T^{\prime} D\right) \\
& \geq\left(\sum_{i \neq j^{\prime}} A_{i}+1\right)-T^{\prime} D^{2} \sum_{j=1}^{N}\left(k_{j}+1\right) .
\end{aligned}
$$

So, we have

$$
\sum_{j=1}^{N}\left(k_{j}+1\right)+1>\frac{Q}{T^{\prime} D^{2}}
$$

If $\sum_{i \neq j^{\prime}} A_{i}>\sum_{j=1}^{N} B_{j}$, we use the first inequality in Case I, together with (2.20) and (2.21), to derive

$$
\begin{aligned}
\sum_{j=1}^{N} B_{j}(Q \gamma+1) & \geq \sum_{i=1}^{M} A_{i} a_{r_{i}}+1>\sum_{i \neq j^{\prime}} A_{i} a_{r_{i}} \\
& >\sum_{i \neq j^{\prime}} A_{i}\left(Q \gamma-\left(k_{i}+1\right) T^{\prime} D \gamma-1\right) \\
& \geq\left(\sum_{j=1}^{N} B_{j}+1\right) Q \gamma-T^{\prime} D \gamma \sum_{i \neq j^{\prime}} A_{i}\left(k_{i}+1\right)-\sum_{i \neq j^{\prime}} A_{i}
\end{aligned}
$$




$$
\geq\left(\sum_{j=1}^{N} B_{j}+1\right) Q \gamma-T^{\prime} D^{2} \gamma \sum_{i \neq j^{\prime}}\left(k_{i}+1\right)-\sum_{i \neq j^{\prime}} A_{i} .
$$

Therefore

$$
T^{\prime} D^{2} \sum_{i \neq j^{\prime}}\left(k_{i}+1\right)+\frac{1}{\gamma}\left(\sum_{i \neq j^{\prime}} A_{i}+\sum_{j=1}^{N} B_{j}\right)>Q .
$$

Similarly to $(2.23)$, we have

$$
\frac{1}{\gamma}\left(\sum_{i \neq j^{\prime}} A_{i}+\sum_{j=1}^{N} B_{j}\right)<T^{\prime} D^{2}(v-1) .
$$

Thus,

$$
\sum_{i \neq j^{\prime}}\left(k_{i}+1\right)+v-1>\frac{Q}{T^{\prime} D^{2}} .
$$

In Case II, similar to Case I, if $\sum_{i \neq j^{\prime}} A_{i}<\sum_{j=1}^{N} B_{j}$, we use the first inequality in Case II, and (2.20) and (2.21), to derive

$$
\sum_{j=1}^{N}\left(k_{j}+1\right)+v>\frac{Q}{T^{\prime} D^{2}} .
$$

If $\sum_{i \neq j^{\prime}} A_{i}>\sum_{j=1}^{N} B_{j}$, we use the second inequality in Case II, and (2.16), to obtain

$$
\sum_{i \neq j^{\prime}}\left(k_{i}+1\right)>\frac{Q}{T^{\prime} D^{2}}
$$

2) $\frac{a_{j^{*}}}{q_{j^{*}}} \in\left\{\frac{a_{s_{j}}}{q_{s_{j}}}: 1 \leq j \leq N\right\}$. Because of symmetry, one can get results similar to the above in this case as well.

In Case I, if $\sum_{i=1}^{M} A_{i}<\sum_{j \neq j^{\prime}}^{N} B_{j}$, we have

$$
\sum_{j \neq j^{\prime}}\left(k_{j}+1\right)>\frac{Q}{T^{\prime} D^{2}} .
$$

If $\sum_{i=1}^{M} A_{i}>\sum_{j \neq j^{\prime}}^{N} B_{j}$, then

$$
\sum_{i=1}^{M}\left(k_{i}+1\right)+v>\frac{Q}{T^{\prime} D^{2}} .
$$

In Case II, if $\sum_{i=1}^{M} A_{i}<\sum_{j \neq j^{\prime}}^{N} B_{j}$, one has

$$
\sum_{j \neq j^{\prime}}\left(k_{j}+1\right)+v-1>\frac{Q}{T^{\prime} D^{2}} .
$$


If $\sum_{i=1}^{M} A_{i}>\sum_{j \neq j^{\prime}}^{N} B_{j}$, then

$$
\sum_{i=1}^{M}\left(k_{i}+1\right)+1>\frac{Q}{T^{\prime} D^{2}}
$$

Similar to (2.27), combining (2.28-2.35), we find that

$$
s-r \geq\left[\frac{Q}{v T^{\prime} D^{2}}\right] \text {, if } \frac{a_{j^{*}}}{q_{j^{*}}} \in\left\{\frac{a_{r_{i}}}{q_{r_{i}}}: 1 \leq i \leq v\right\} .
$$

Thus, for $t=1$ and $q_{j^{*}}<T^{\prime} D$, by (2.15), (2.27), and (2.36), we get,

$$
s-r \geq\left[\frac{Q}{v T^{\prime} D^{2}}\right] \text {. }
$$

Therefore, by (2.9), (2.11), (2.12), and (2.37), there exists a constant $C=$ $C(v)$, such that

$$
s-r>\frac{Q}{C D^{2}}
$$

We now provide an admissible choice for the constant $C(v)$ in our theorem.

Take $T=16$, and $T^{\prime}=4 v$. In case $t=0$, by (2.9), we get

$$
s-r>\frac{Q}{32 v D} \text {. }
$$

In case $t \geq 2$, by (2.11), we get

$$
s-r>\frac{Q}{64 v D}
$$

In case $t=1$, and $q_{j^{*}} \geq T^{\prime} D$, by (2.12), we obtain

$$
s-r>\frac{Q}{D}\left(\frac{1}{32 v}-\frac{1}{60 v}\right)>\frac{Q}{69 v D} .
$$

In case $t=1$, and $q_{j^{*}}<T^{\prime} D$, by (2.37), we get

$$
s-r \geq\left[\frac{Q}{4 v^{2} D^{2}}\right] \text {. }
$$

Hence, one can take $C=C(v)=69 v^{2}$.

This completes the proof of the theorem.

\section{Examples}

In this section we provide counterexamples in some cases when one does not assume the conditions on coefficients from the statement of the theorem. First, we assume that

$$
\sum_{i=1}^{v} A_{i}=0, \text { i.e., } \sum_{i=1}^{M} A_{i}=\sum_{j=1}^{N} B_{j} .
$$


Consider the neighbors of $\frac{1}{2}$. If $Q$ is even, let $Q=2 K$, then the neighbors of $\frac{1}{2}$ are

$$
\ldots, \frac{K-2}{2 K-3}, \frac{K-1}{2 K-1}, \frac{1}{2}, \frac{K}{2 K-1}, \frac{K-1}{2 K-3}, \ldots
$$

Let $\gamma_{0}=\frac{1}{2}$, and let $\gamma_{-i}(i>0)$ be the $i$-th fraction to the left of $\frac{1}{2}$, and $\gamma_{i}$ be the $i$-th fraction to the right of $\frac{1}{2}$.

Then, we have the following examples.

Example 1. Let $v=3$ and $D=2$. Denote as usual $\mathbf{x}=\left(x_{1}, x_{2}, x_{3}\right)$, and consider the linear form

$$
L(\mathbf{x})=x_{1}+x_{2}-2 x_{3} .
$$

Take the fractions $\gamma_{-2}, \gamma_{-1}$, and $\gamma_{2}$,

$$
\gamma_{-2}=\frac{K-2}{2 K-3}, \gamma_{-1}=\frac{K-1}{2 K-1}, \gamma_{2}=\frac{K-1}{2 K-3}
$$

Then,

$$
L(\mathbf{a})=(K-2)+(K-1)-2(K-1)=-1,
$$

and

$$
L(\mathbf{q})=(2 K-3)+(2 K-1)-2(2 K-3)=2 .
$$

So, for any even $Q$, we always have

$$
L(\mathbf{a}) L(\mathbf{q})<0 .
$$

But, here $s-r=4$. Thus, there does not exist a constant $C$ such that, for any $Q$ and any three fractions satisfying $s-r \leq \frac{Q}{C D^{2}}$, we have

$$
L(\mathbf{a}) L(\mathbf{q}) \geq 0 .
$$

Actually, for any two positive integers $m$ and $n$ satisfying $m+n \leq D$, one may consider the linear form

$$
L(\mathbf{x})=m x_{1}+n x_{2}-(m+n) x_{3} .
$$

Then taking the same three fractions $\gamma_{-2}, \gamma_{-1}$, and $\gamma_{2}$, as above, one sees that

$$
L(\mathbf{a})=m(K-2)+n(K-1)-(m+n)(K-1)=-m,
$$

and

$$
L(\mathbf{q})=m(2 K-3)+n(2 K-1)-(m+n)(2 K-3)=2 n .
$$

Thus, we always have

$$
L(\mathbf{a}) L(\mathbf{q})<0 .
$$

Example 2. Let $v=4, D=4$, and consider the linear form

$$
L(\mathbf{x})=x_{1}+x_{2}+2 x_{3}-4 x_{4} .
$$

Take the fractions

$$
\gamma_{-3}=\frac{K-3}{2 K-5}, \gamma_{-2}=\frac{K-2}{2 K-3}, \gamma_{-1}=\frac{K-1}{2 K-1}, \gamma_{2}=\frac{K-1}{2 K-3} .
$$


Here $s-r=5$. But,

$$
L(\mathbf{a})=(K-3)+(K-2)+2(K-1)-4(K-1)=-3,
$$

and

$$
L(\mathbf{q})=(2 K-5)+(2 K-3)+2(2 K-1)-4(2 K-3)=2 .
$$

Thus, $L(\mathbf{a}) L(\mathbf{q})<0$. Hence, there is no constant $C$ such that, for any four fractions, if $s-r \leq \frac{Q}{C D^{2}}$, we have

$$
L(\mathbf{a}) L(\mathbf{q}) \geq 0 .
$$

Example 3. Let $v=5, D=2$, and consider the linear form

$$
L(\mathbf{x})=x_{1}+x_{2}+2 x_{3}-2 x_{4}-2 x_{5} .
$$

Let us choose the fractions

$\gamma_{-3}=\frac{K-3}{2 K-5}, \gamma_{-2}=\frac{K-2}{2 K-3}, \gamma_{-1}=\frac{K-1}{2 K-1}, \gamma_{2}=\frac{K-1}{2 K-3}, \gamma_{3}=\frac{K-2}{2 K-5}$.

Then,

$$
L(\mathbf{a})=(K-3)+(K-2)+2(K-1)-2(K-1)-2(K-2)=-1,
$$

and

$$
L(\mathbf{q})=(2 K-5)+(2 K-3)+2(2 K-1)-2(2 K-3)-2(2 K-5)=6 .
$$

Thus, for any even $Q, L(\mathbf{a}) L(\mathbf{q})<0$. Here $s-r=6$.

Example 4. Let $v=6, D=3$, and consider the linear form

$$
L(\mathbf{x})=x_{1}+2 x_{2}+3 x_{3}-2 x_{4}-x_{5}-3 x_{6} .
$$

Take the fractions

Then,

$$
\begin{gathered}
\gamma_{-3}=\frac{K-3}{2 K-5}, \gamma_{-2}=\frac{K-2}{2 K-3}, \gamma_{-1}=\frac{K-1}{2 K-1}, \\
\gamma_{1}=\frac{K}{2 K-1}, \gamma_{2}=\frac{K-1}{2 K-3}, \gamma_{3}=\frac{K-2}{2 K-5} .
\end{gathered}
$$

$$
L(\mathbf{a})=(K-3)+2(K-2)+3(K-1)-2 K-(K-1)-3(K-2)=-3,
$$

and

$L(\mathbf{q})=(2 K-5)+2(2 K-3)+3(2 K-1)-2(2 K-1)-(2 K-3)-3(2 K-5)=6$.

Thus, for any even $Q, L(\mathbf{a}) L(\mathbf{q})<0$. Here $s-r=6$.

Note. If $Q$ is odd, $Q=2 K+1$ say, then the neighbors of $\frac{1}{2}$ are

$$
\ldots, \frac{K-1}{2 K-1}, \frac{K}{2 K+1}, \frac{1}{2}, \frac{K+1}{2 K+1}, \frac{K}{2 K-1}, \ldots
$$

Then, the above linear forms also provide counterexamples in this case. We remark that one can also construct counterexamples using the neighbors of $\frac{1}{3}$.

Acknowledgement. The authors are grateful to the referee for many useful comments and suggestions. 


\section{References}

[1] E. Alkan, M. S. Xiong, and A. Zaharescu, A bias phenomenon on the behavior of Dedekind sums, Math. Res. Lett. 15 (2008), no. 5, 1039-1052.

2] — Quotients of values of the Dedekind eta function, Math. Ann. 342 (2008), no. $1,157-176$.

[3] J. Athreya and Y. Cheung, A Poincaré section for horocycle flow on the space of lattices, to appear in Int. Math. Res. Not. IMRN 2013.

[4] F. P. Boca, C. Cobeli, and A. Zaharescu, Distribution of lattice points visible from the origin, Comm. Math. Phys. 213 (2000), no. 2, 433-470.

5] _ A conjecture of R. R. Hall on Farey points, J. Reine Angew. Math. 535 (2001), 207-236.

6] F. P. Boca and A. Zaharescu, Farey fractions and two-dimensional tori, Noncommutative geometry and number theory, 57-77, Aspects Math., E37, Vieweg, Wiesbaden, 2006.

[7] P. Erdös, A note on Farey series, Quart. J. Math., Oxford Ser. 14 (1943), 82-85.

[8] G. H. Hardy, J. E. Littlewood, and G. Pólya, Inequalities, Cambridge Univ. Press, Cambridge, 1988, Reprint of the 1952 edition. Cambridge Mathematical Library, xii+324pp.

[9] G. H. Hardy and E. M. Wright, An Introduction to the Theory of Numbers, Clarendon Press, Oxford, 1938.

[10] A. E. Mayer, A mean value theorem concerning Farey series, Quart. J. Math., Oxford Ser. 13 (1942), 48-57.

[11] _ On neighbours of higher degree in Farey series, Quart. J. Math., Oxford Ser. 13 (1942), 185-192.

[12] A. Zaharescu, The Mayer-Erdös phenomenon, Indag. Math. (N.S.) 17 (2006), no. 1, $147-156$.

XiAnCHANG MENG

Department of Mathematics

UNIVERSiTY OF ILLiNOIS AT URBANA-CHAMPAIGN

1409 W. Green Street, Urbana, Illinois 61801, USA

E-mail address: xmeng13@illinois.edu

Alexandru Zaharescu

Department of Mathematics

UNIVERSITY OF ILLINOIS AT URBANA-CHAMPAIGN

1409 W. Green Street, Urbana, Illinois 61801, USA

E-mail address: zaharesc@math.uiuc.edu 